\title{
Characterization of counter-rotating streamwise vortices in flat rectangular channel with one-sided wavy wall
}

\author{
Yann Bouremel ${ }^{a, 1, *}$, Hatsari Mitsudharmadi ${ }^{\mathrm{b}}$, Alexander C. Budiman ${ }^{\mathrm{c}}$, \\ Sonny H. Winoto ${ }^{\mathrm{d}, 2}$ \\ ${ }^{a}$ Temasek Laboratories, National University of Singapore, Singapore 117411, Singapore \\ ${ }^{b}$ Clean Combustion Research Center, King Abdullah University of Science and \\ Technology, Thuwal 23955-6900, Kingdom of Saudi Arabia \\ ${ }^{c}$ Department of Mechanical Engineering, National University of Singapore, Singapore \\ 117576, Singapore \\ ${ }^{d}$ Faculty of Engineering, Universitas Diponegoro, Tembalang Campus, Semarang 50275, \\ Indonesia
}

\section{Abstract}

Particle Image Velocimetry (PIV) has been used to characterize the evolution of counter-rotating streamwise vortices in a rectangular channel with one sided wavy surface. The vortices were created by a uniform set of saw-tooth carved over the leading edge of a flat plate at the entrance of a flat rectangular channel with one-sided wavy wall. PIV measurements were taken over the spanwise and streamwise planes at different locations and at Reynolds number of 2500. Two other Reynolds numbers of 2885 and 3333 have also been considered for quantification purpose. Pairs of counter-rotating streamwise vortices have been shown experimentally to be centered along the spanwise

\footnotetext{
${ }^{*}$ Corresponding author

Email address: y.bouremel@ucl.ac.uk (Yann Bouremel )

${ }^{1}$ Present address: National Institute for Health Research (NIHR) Biomedical Research Centre at Moorfields Eye Hospital NHS Foundation Trust and UCL Institute of Ophthalmology, London, UK

${ }^{2}$ Formely at: Department of Mechanical Engineering, National University of Singapore, Singapore 117576, Singapore
} 
direction at the saw-tooth valley where the vorticity $\omega_{z}=0$. It has also been found that the vorticity $\omega_{z}$ of the pairs of counter-rotating vortices decreases along the streamwise direction, and increases with the Reynolds number. Moreover, different quantifications of such counter-rotating vortices have been discussed such as their size, boundary layer, velocity profile and vorticity. The current study shows that the mixing due to the wall shear stress of counter-rotating streamwise vortices as well as their averaged viscous dissipation rate of kinetic energy decrease over flat and adverse pressure gradient surfaces while increasing over favourable pressure gradient surfaces. Finally, it was also demonstrated that the main direction of stretching is orientated at around $45^{\circ}$ with the main flow direction.

Keywords: Counter-rotating vortices, Wavy wall, Vorticity, Mixing, Stretching

\section{Introduction}

Wavy walls are used for heat and mass transfer for flows of high Peclet numbers (Nishimura et al., 1986, 1985). One of the very first work from Clauser and Clauser (1937) noticed that laminar flows transition to turbulence at higher Reynolds numbers on flat and convex surfaces compared to concave surfaces. The fully developed laminar and turbulent flows in a wavy channel have been characterized experimentally as well as numerically with one of the earlier work using flow visualization techniques such as hydrogen bubble and laser Doppler anemometry to look at vortices in water channel with $90^{\circ}$ bend (Winoto and Crane, 1980; Nishimura et al., 1985, 1986, 1990; Floryan, 2002;

Bahaidarah et al., 2005; Asai and Floryan, 2006; Aider et al., 2009; Winoto 
et al., 2011; Tandiono et al., 2013). Flow in a channel over wavy walls can become turbulent due to instability of the shear layer caused by the main flow interacting with a Kelvin-Helmhotz type vortex or a centrifugal instability over concave surface (H.Görtler, 1954; Smith, 1955; Gschwind et al., 1995) which takes the form of counter-rotating streamwise vortices called Görtler vortices with a form of a symmetrical mushroom-like shape over its cross section (Bakchinov et al., 1995; Mitsudharmadi et al., 2004, 2006; Tandiono et al., 2009; Winoto et al., 2011; Budiman et al., 2015). There are two regions in the flowfield of these vortices: the so-called upwash region where low velocity fluid is lifted from the surface and the so-called downwash region where higher velocity fluid is pushed back towards the surface resulting in thinner boundary layer and higher shear stress (Winoto et al., 2011).

It has been found that the amplitude as well as the wavelength of the wavy surface are critical for whether the counter-rotating vortices can be formed regardless of the Reynolds number while convex parts tend to prevent the formation of such vortices and stabilise the flow (Floryan, 2002). The stability of counter-rotating vortices inside a wavy channel has been quantified by Floryan (2007) with the following two equations (1a) and (1b):

$$
\begin{aligned}
\ln \left(R e_{\text {crit }}\right) & =-1.3205 \ln (S)+1.8888 \\
S & =\frac{a}{H}
\end{aligned}
$$

where $a$ is the amplitude of the wavy surfaces and $H$, the channel gap. 
If the Reynolds number, $R e$, as defined by:

$$
R e=\frac{1}{2} \frac{U_{\infty} H}{\nu}
$$

where $U_{\infty}$ being the free-stream velocity and $\nu$ the fluid kinematic viscosity is

below $R e_{c r i t}$, then the flow is stable and counter-rotating streamwise vortices cannot be generated.

Most of the reports mentioned earlier characterise fully-developed channel flow in laminar or turbulent regime. However the discussion about boundarylayer laminar flow in channel is still quite rare in the literature. This current study aims to characterize the induced counter-rotating streamwise vortices which evolve in channel flow with one-sided corrugated wall. Recently, Budiman et al. (2016) discussed the development of such vortices by means of hot-wire anemometry. However the report is only limited to the spanwise $y-z$ plane. This paper is intended to complement that report by focusing on the streamwise $x-y$ and $x-z$ planes, together with discussions on mixing properties that have not been covered by Budiman et al. (2016).

\section{Experimental set-up}

A zigzag pattern of uniform saw-tooth is crafted on the leading edge of a 150 $\mathrm{mm}$ flat plate attached to a corrugated (wavy) plate. Air flowing through this set of saw-tooth leads to the creation of uniform spanwise wavelength counter-rotating streamwise vortices. Each tooth depth is $6.3 \mathrm{~mm}$ with a spanwise wavelength of $15 \mathrm{~mm}$. The wavy plate has a corrugation amplitude 
$a=3.75 \mathrm{~mm}$ with a wavelength $\lambda=76 \mathrm{~mm}$. The channel gap $H$ was set at $40 \mathrm{~mm}$ from the flat plate as shown in Fig. 1.

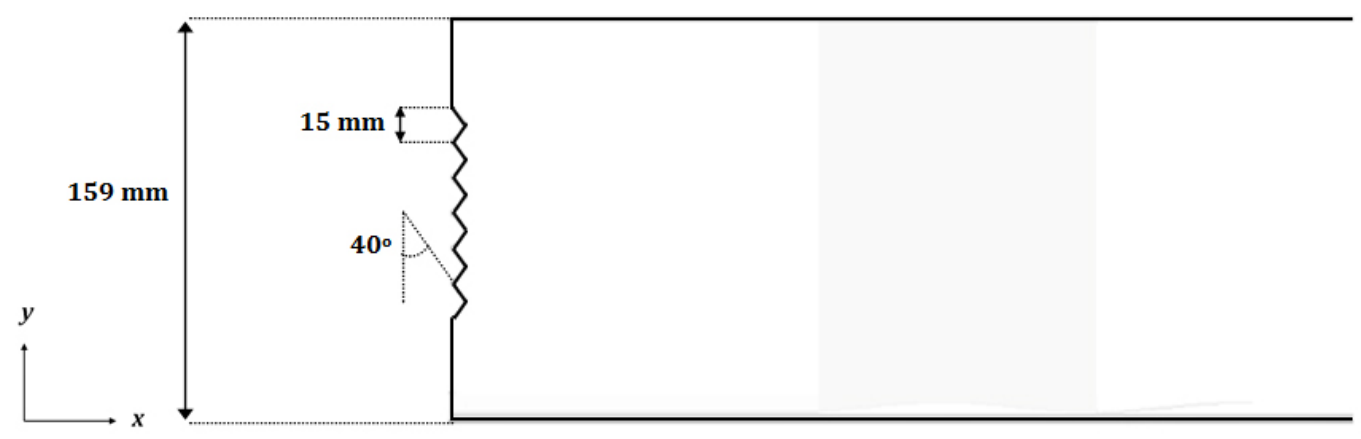

(a)

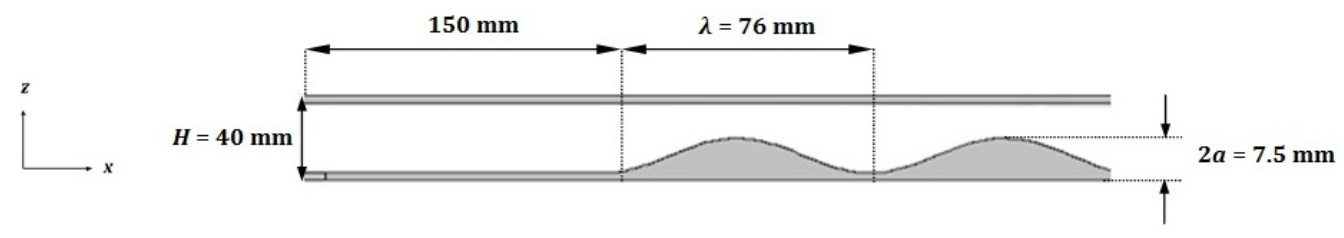

(b)

Figure 1: Top view (a) and side view (b) of one-sided wavy wall channel.

Flow-field velocity measurements were carried out by using a two-velocitycomponent Particle Image Velocimetry (PIV) system. The flow was uniformly seeded upstream of the wind-tunnel air intake with olive oil particles from a DANTEC 10F03 seeding generator. The droplets produced were in the average size Sauter mean diameter (SMD) 2-5 $\mu \mathrm{m}$ whose reflections correspond to no more than 3 pixels in the captured images which allows a good resolution of the particle displacement when cross correlation methods are adopted. A dual-head Litron DualPower 200-15 Nd:YAG laser operating at the second harmonic $(532 \mathrm{~nm})$ at approximately $150 \mathrm{~mJ}$ per pulse was 
used in conjunction with sheet-forming optics to form a thin sheet $(\approx 1 \mathrm{~mm})$. The images were acquired using double frame mode by a $2048 \times 2048$ pixels HiSense 620 camera with a Zeiss $50 \mathrm{~mm} \mathrm{f} / 2.0$ macro lens $\left(125 \times 125 \mathrm{~mm}^{2}\right.$ field of view for the $x-y$ plane and $46 \times 46 \mathrm{~mm}^{2}$ for the $x-z$ plane). The resulting resolution is approximately 61 and $22 \mu \mathrm{m}$ per pixel for the $x-y$ and $x-z$ plane, respectively. The camera viewed the streamwise laser sheet orthogonally over the entire field of view. A computer with dual Intel Core processors was used for data acquisition. The acquired frames were divided into $32 \times 32$ pixel and $16 \times 16$ interrogation windows which contain at least 3 seeding particles for the $x-y$ and the $x-z$ plane, respectively. Based on the flow velocity and the size of the interrogation area, the time separation between the two laser flashes (double frame mode) was set such that the maximum displacement of a particle is no more than $25 \%$ of the interrogation size which is the optimum displacement for the DANTEC software to calculate accurately the particle velocity. This time separation varied from $130 \mu \mathrm{s}$ to $650 \mu \mathrm{s}$ depending on the resolution and the location of the field of view. For each frame, subregions were adaptatively cross-correlated using multi-pass processing with a final $50 \%$ overlap that gives a final interrogation area of $16 \times 16$ pixels after processing for the $x-y$ plane and $8 \times 8$ pixels for the $x-z$ plane. The resulting vector fields were post-processed to remove remaining spurious vectors. This arrangement gives a velocity vector grid of $127 \times 127$ points for the $x-y$ plane and $255 \times 255$ points for the $x-z$ plane which translates to velocity vectors separated by about $1 \mathrm{~mm}$ over the $x-y$ field of view and $0.18 \mathrm{~mm}$ over the $x-z$ field of view. For each acquisition, 500 velocity-vector images were taken for statistical averaging at a trigger 
rate of $7 \mathrm{~Hz}$.

The Reynolds number Re was set at 2500, 2885 and 3333 and according to Equations (1a) and (1b): $R e_{\text {crit }}=150.6$ and $S=0.094$, respectively. According to Floryan's global instability diagram (Floryan, 2007), the experimental condition set were within the instability region where the formation of counter-rotating streamwise vortices can be observed.

\section{Results and Discussion}

\subsection{PIV results along the $(x, y)$ plane}

In Fig. 2 (a) - (c), the vorticity contour plots at $R e=2500$ for different heights from $z=2$ to $7 \mathrm{~mm}$ for $x=0 \mathrm{~mm}$ to $125 \mathrm{~mm}$ are presented. It should be noted that this section is part of the flat plate. Indeed, the distance from the surface $(\mathrm{z})$ in the wavy part, and thus the wall effect, varies along the contour plot and therefore no conclusions can be given. It was decided to first study the flat part where the distance from the wall $(z)$ is fixed before presenting results over the wavy part. In these figures, the position of the teeth that pre-set the uniform spacing between counter rotating streamwise vortices are indicated by a saw-tooth in black located at the origin of the figure. This origin will be used throughout this article.

In Fig. 2 (a) - (c), it can be seen that 3 pairs of counter-rotating vortices are created along the streamwise direction. Each pair is centered at $y=7.3$ $\mathrm{mm}, 22.3 \mathrm{~mm}$ and $37.3 \mathrm{~mm}$, which correspond exactly to the spanwise lo- 
cations of the saw-tooth trough. It indicates the variation of the boundary layer thickness along the spanwise direction as the counter rotating streamwise vortices are formed and evolve downstream. From $x=0 \mathrm{~mm}$ to $19 \mathrm{~mm}$, the vorticity levels from each plot in Fig. 2 (a) - (c) are equal to zero because of a limitation of the experimental set-up. Indeed, the channel was locally opaque at that location. Therefore, the seeding particles could not be seen through this section with the camera, and no velocity vectors could be calculated at these locations leading to a zero vorticity region. This zero-vorticity region will be present in each contour plot over the $(x, y)$ plane.

It can be noted that along the streamwise direction the vorticity level of each vortex decreases as depicted by the change of colours from dark red/blue to bright red/blue, for example at $y=38.6 \mathrm{~mm}$, the vorticity level varies from $-259 \mathrm{~s}^{-1}$ to $-127 \mathrm{~s}^{-1}$ from $x=23.8 \mathrm{~mm}$ to $121.7 \mathrm{~mm}$ along the flat plate in Fig. 2 (a). When the height $z$ is increased by $2 \mathrm{~mm}$ from Fig. 2 (a) to Fig. 2 (b) the vorticity level of each counter-rotating vortices increases. For example at $x=81.7 \mathrm{~mm}$ and $y=39.6 \mathrm{~mm}, \omega_{z}=-123 \mathrm{~s}^{-1}$ at $z=2 \mathrm{~mm}$ in Fig. 2 (a) and $-144 \mathrm{~s}^{-1}$ at $z=4 \mathrm{~mm}$ in Fig. 2 (b). In Fig. 2 (c), the vorticity level $\omega_{z}=0$ across most of the flow showing that at this location, the $(x, y)$ plan selected is located in the vicinity of the maximum speed in the Poiseuille velocity profile. 


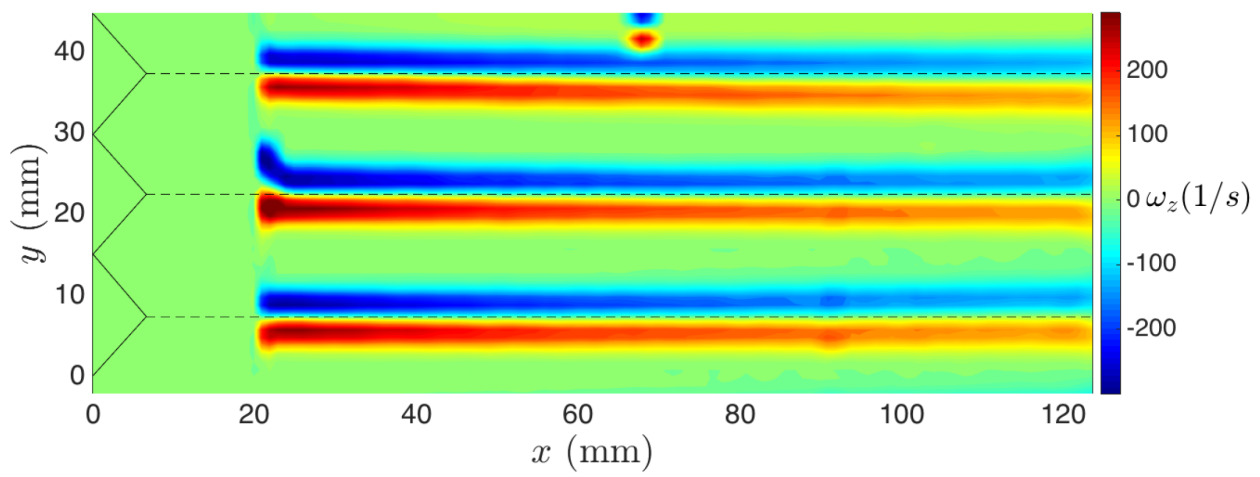

(a)

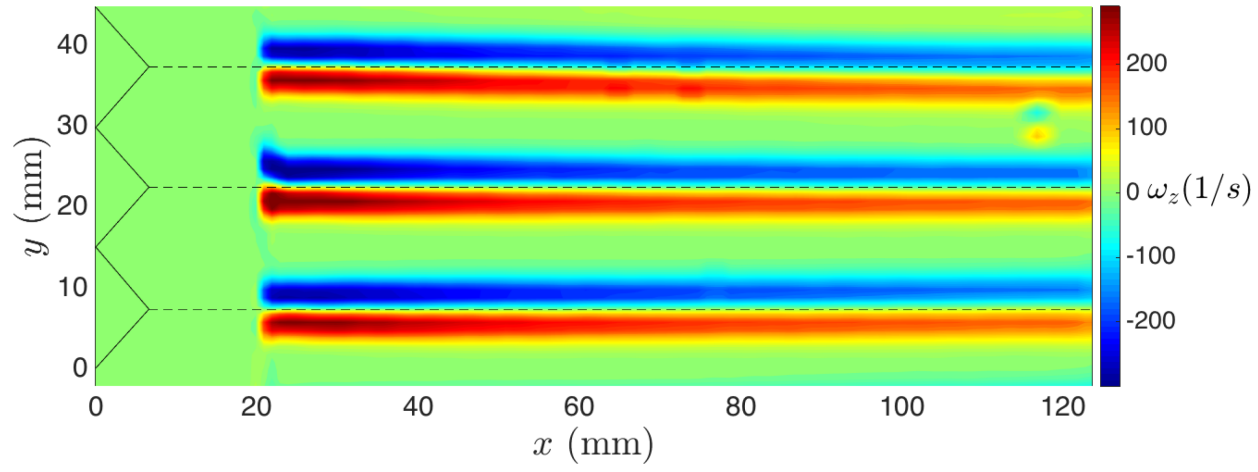

(b)

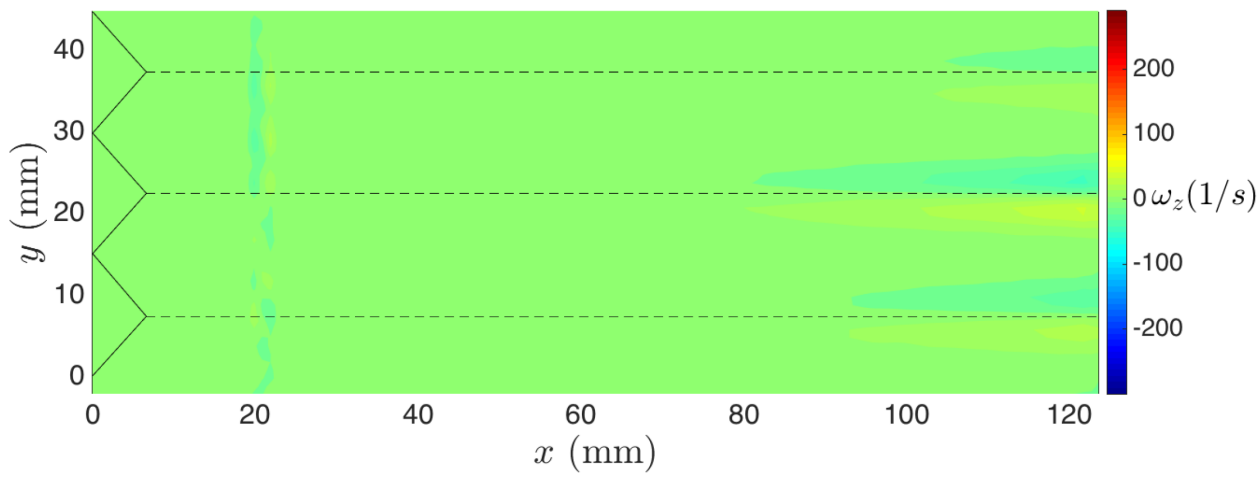

(c)

Figure 2: Wall-normal vorticity $\left(\omega_{z}\right)$ contours at $R e=2500$ along planes located at (a): $z=2 \mathrm{~mm},(\mathrm{~b}): z=4 \mathrm{~mm}$ and (c): $z=7 \mathrm{~mm}$. 
In Fig. 3 (a), the evolutions of the absolute averaged vorticity maximum of 3 pairs of counter-rotating vortices are calculated along the streamwise direction at $z=2 \mathrm{~mm}$ for $R e=2500,2885$ and 3333. It can be seen in Fig. 3 (a) that the absolute maximum vorticity level decreases along the streamwise direction $\vec{x}$ while increasing with the Reynolds number. It can be understood that this decrease of $\bar{\omega}_{z_{\max }}$ can be attributed to the lifting of the vortex cores as the stem of the mushroom-like structures grows, similarly to the visualisation results in Fig. 11 reported by Budiman et al. (2015).

In Fig. 3 (b) - (d), the evolutions of the absolute averaged vorticity maximum level obtained similarly to Fig. 3 (a) are plotted for $z=2 \mathrm{~mm}$ and $4 \mathrm{~mm}$ for $R e=2500,2885$ and 3333, respectively. It can be seen in Fig. 3 (b) at $R e=2500$, that $\omega_{z_{\max }}$ when averaged along the streamwise direction from $x=22 \mathrm{~mm}$ to $125 \mathrm{~mm}$ is equal to $153 \mathrm{~s}^{-1}$ at $z=2 \mathrm{~mm}$ while at $z=4$ $\mathrm{mm}$, over the same range along the streamwise direction, $\overline{\omega_{z_{\max }}}=168 \mathrm{~s}^{-1}$, hence an increase of approximately 10\%. In Fig. 3 (c), at $R e=2885$, from $x=22 \mathrm{~mm}$ to $125 \mathrm{~mm} \overline{\omega_{z_{\max }}}=186 \mathrm{~s}^{-1}$ at $z=2 \mathrm{~mm}$ and $\overline{\omega_{z_{\max }}}=177 \mathrm{~s}^{-1}$ at $z=4 \mathrm{~mm}$, a slight decrease of around $5 \%$. Finally at $R e=3333$, when averaging over the same range along the streamwise direction, $\overline{\omega_{z_{\max }}}=221$ $\mathrm{s}^{-1}$ at $z=2 \mathrm{~mm}$ and $\overline{\omega_{z_{\max }}}=167 \mathrm{~s}^{-1}$ at $z=4 \mathrm{~mm}$, a decrease close to $25 \%$. Therefore, at $R e=2500, \overline{\omega_{z_{\max }}}$ increases with $z$ showing that the vortex cores are located above $z=2 \mathrm{~mm}$ then as the Reynolds number increases $\overline{\omega_{z_{\max }}}$ decreases with $z$ in a low manner for $R e=2885$ (decrease of $5 \%$ ) and more significantly at $R e=3333$ (around 25\%) meaning that in both cases the location $z=4 \mathrm{~mm}$ is further away from the vortex cores than $z=2$ 
$\mathrm{mm}$. These show that as Re increases, the pair of counter-rotating vortices are getting smaller in size and closer to the plate. It implies that the higher the Reynolds number is, the more stable the flow will be as smaller vortices are generated.

From Fig. 3 (a) - (d), it can be shown that the vorticity and the size of the counter-rotating streamwise vortices are sensitive to the Reynolds number. However, for each Reynolds number, the evolution of the vorticity $\overline{\omega_{z_{\max }}}$ is similar along the streamwise direction namely a similar decrease with $\vec{x}$. Therefore, in the rest of the article, parameters such as vorticity, kinetic energy dissipation, velocity profile and parameters akin boundary layers are studied only for $R e=2500$. 


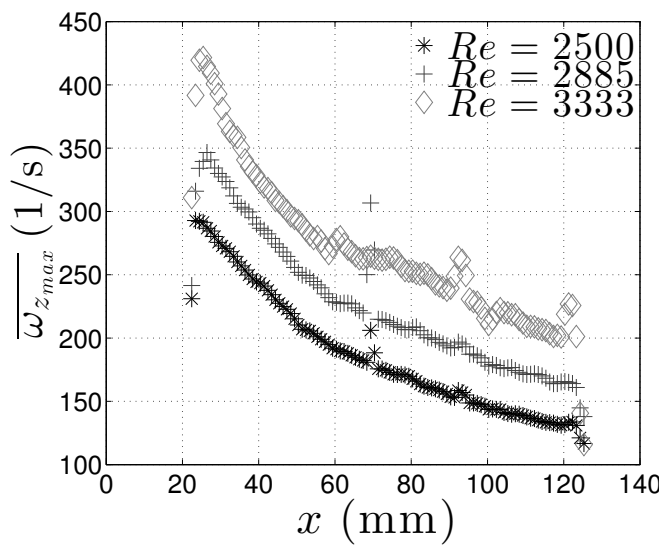

(a)

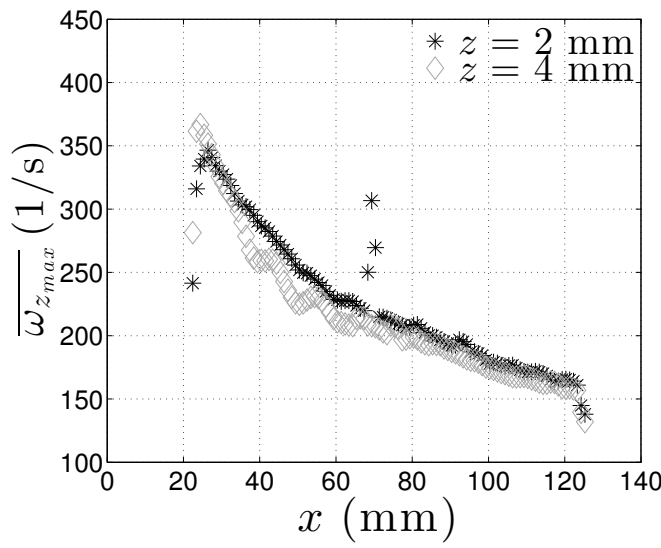

(c)

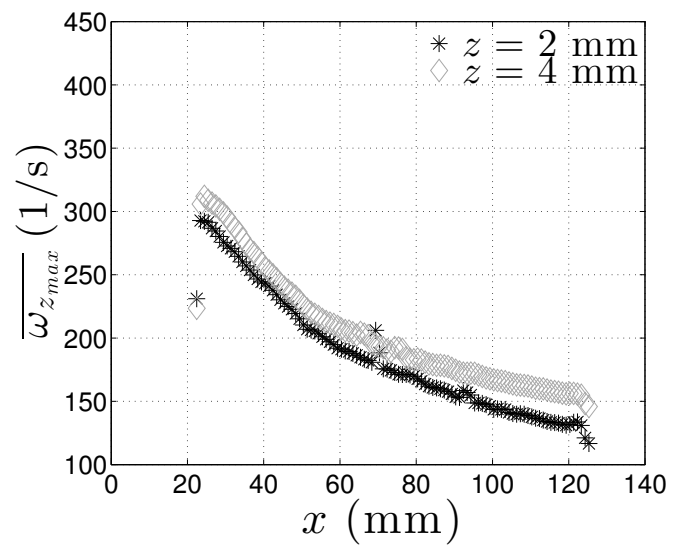

(b)

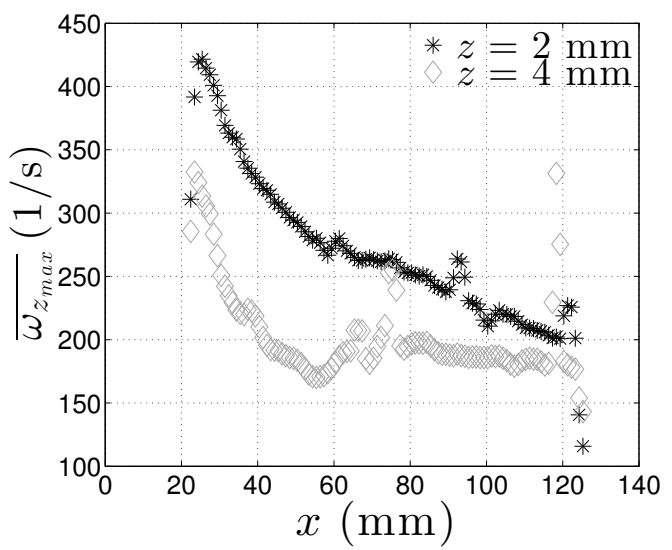

(d)

Figure 3: Evolution of the absolute averaged wall-normal vorticity maximum $\left(\overline{\omega_{z_{\max }}}\right)$ along the streamwise direction, (a): at $z=2 \mathrm{~mm}$ for $R e=2500, R e=2885$ and $R e=3333$; (b): at $z=2$ and $4 \mathrm{~mm}$ for $R e=2500$; (c): at $z=2$ and $4 \mathrm{~mm}$ for $R e=2885$; (d): at $z=2$ and $4 \mathrm{~mm}$ for $R e=3333$.

Vorticity profiles along the spanwise direction at different streamwise locations for $z=4 \mathrm{~mm}$ are plotted in Fig. 4 for $R e=2500$. Similarly to Fig. 3 (a) - (d) where $\overline{\omega_{z_{\max }}}$ decreases along the streamwise direction, it can be noted that the different profiles of $\omega_{z}$ obtained along $y$ exhibit lower values 


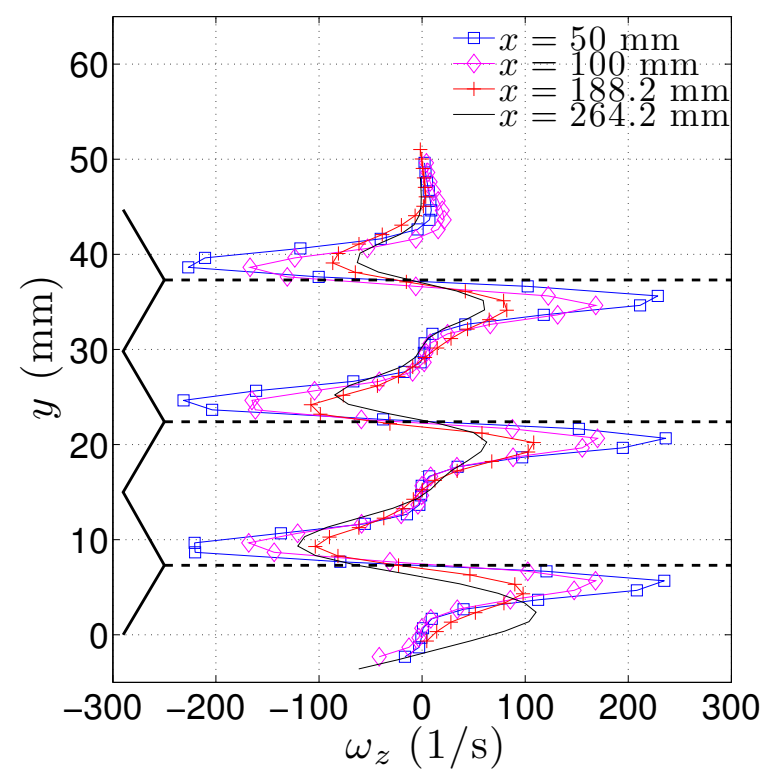

Figure 4: Wall-normal vorticity $\left(\omega_{z}\right)$ profile plots at different streamwise locations along planes located at $z=4 \mathrm{~mm}$ for $R e=2500$.

with $\vec{x}$. For example, at $y=35.6 \mathrm{~mm}, \omega_{z}=229 \mathrm{~s}^{-1}, 169 \mathrm{~s}^{-1}, 82 \mathrm{~s}^{-1}$ and $60 \mathrm{~s}^{-1}$ for $x=50 \mathrm{~mm}, 100 \mathrm{~mm}, 188.2 \mathrm{~mm}$ and $264.2 \mathrm{~mm}$, respectively. In Fig. 4, the regions where the vorticity goes from negative to positive as y increases corresponding to the downwash region are clearly defined at $x=50$ $\mathrm{mm}$ showing an inflexion point (approximately at $y=0,15$, and $30 \mathrm{~mm}$ ). As $x$ increases, the width of this inflexion point reduces and is not present anymore at $x=264.2 \mathrm{~mm}$ showing that the different pairs of counter-rotating vortices are getting closer to each other along $x$ prior to its breakdown as they grow in size while their vorticity levels, $\omega_{z}$, decrease.

To study the role of the vortices in a mixing process, the viscous dissipation rate of kinetic energy along the $(x, y)$ plane, $\epsilon_{x y}$, is plotted at $z=4 \mathrm{~mm}$ 
for $R e=2500$ in Fig. 5:

$$
\epsilon_{x y}=\nu \frac{\partial u_{j}}{\partial x_{i}}\left(\frac{\partial u_{i}}{\partial x_{j}}+\frac{\partial u_{j}}{\partial x_{i}}\right)
$$

with the indices $i=x, y$ and $j=x, y$ denoting the Einstein summation convention. $\epsilon_{x y}$ is the rate of dissipation of kinetic energy into heat that promotes mixing in the plane $(x, y)$.

The higher $\epsilon_{x y}$ is, the more mixing is occurring in the flow. It can be shown that $\epsilon_{x y}$ decreases along the streamwise direction showing that mixing is decreasing as the counter-rotating vortices travel along $x$. For example for $y=39.6 \mathrm{~mm}, \epsilon_{x y}=0.95 \mathrm{~m}^{2} \mathrm{~s}^{-3}$ at $x=32.8 \mathrm{~mm}$ while at $x=121.7 \mathrm{~mm}$, $\epsilon_{x y}=0.19 \mathrm{~m}^{2} \mathrm{~s}^{-3}$. This is in agreement with the observations from Fig. 2 on the vorticity level decrease of each vortex: as the flow evolves along the streamwise direction, it dissipates kinetic energy hence a decrease as well in the amount of the viscous dissipation rate of kinetic energy in the $(x, y)$ plane. A second parameter important to characterise mixing is the rate-of-deformation tensor since this parameter helps to understand how the flow field deforms particles/drops locally, increasing their interfacial areas before diffusion and complete mixing (Bouremel et al., 2009). Viscous stresses cause deformations of the fluid elements that are determined by spatial variations of local velocities. $\boldsymbol{\nabla} \boldsymbol{u}$ is called the rate-of-deformation tensor, which can be decomposed into a pure rate-of-deformation tensor, also denoted as the rate-of-strain tensor, and a pure rate-of-rotation tensor, as shown in Equation (4):

$$
\frac{\partial u_{i}}{\partial x_{j}}=\frac{1}{2}\left(\frac{\partial u_{i}}{\partial x_{j}}+\frac{\partial u_{j}}{\partial x_{i}}\right)+\frac{1}{2}\left(\frac{\partial u_{i}}{\partial x_{j}}-\frac{\partial u_{j}}{\partial x_{i}}\right)=\boldsymbol{S}_{i j}+\boldsymbol{\Omega}_{i j}
$$

with $\boldsymbol{S}_{i j}$ being the rate-of-strain tensor and $\boldsymbol{\Omega}_{i j}$ being the rate-of-rotation tensor. An improved understanding of the rate-of-deformation tensor can 
be obtained by adopting a coordinate system which is locally directed as the principal axes of the tensor $\boldsymbol{S}_{i j}$. This change of coordinate reported in Equation (5) allows to visualise and quantify the main directions of stretching and compression (Davidson, 2004):

$$
\boldsymbol{\nabla} \boldsymbol{u}=\left(\begin{array}{ccc}
S_{11}^{*} & 0 & 0 \\
0 & S_{22}^{*} & 0 \\
0 & 0 & S_{33}^{*}
\end{array}\right)+\frac{1}{2}\left(\begin{array}{ccc}
0 & -\omega_{3}^{*} & \omega_{2}^{*} \\
\omega_{3}^{*} & 0 & -\omega_{1}^{*} \\
-\omega_{2}^{*} & \omega_{1}^{*} & 0
\end{array}\right)
$$

with $S_{11}^{*}, S_{22}^{*}, S_{33}^{*}$ being the eigenvalues of $S_{i j}$, and $\omega_{1}^{*}, \omega_{2}^{*}, \omega_{3}^{*}$ being the components of the vorticity along the principal axes of $\boldsymbol{S}_{i j}$ in each point. Each $S_{i i}^{*}$ and the corresponding eigenvector define the local intensity and direction of stretching or compression; if $S_{i i}^{*}>0$, the fluid element considered is stretched, whereas if $S_{i i}^{*}<0$, the fluid element is compressed.

Furthermore, according to this coordinate system, the instantaneous viscous dissipation rate of kinetic energy, $\epsilon$, can be expressed in terms of the stretching and compression eigenvalues, $S_{i i}^{*}$, as shown in Equation (6) (Davidson, 2004):

$$
\epsilon=2 \nu \boldsymbol{S}_{\boldsymbol{i j}} \boldsymbol{S}_{\boldsymbol{i j}}=2 \nu\left(S_{11}^{* 2}+S_{22}^{* 2}+S_{33}^{* 2}\right)
$$

Stretching and compression directions are always perpendicular to each other, therefore in Fig. 5, only the stretching directions are reported in white. The eigenvectors are obtained from the strain-rate tensor obtained in 2 dimensions in the $(x, y)$ plane from Equation (5) rewritten in 2 dimensions as:

$$
\boldsymbol{S}_{\boldsymbol{i j}}=\left(\begin{array}{cc}
S_{11}^{*} & 0 \\
0 & S_{22}^{*}
\end{array}\right)
$$

It can be seen that the directions of stretching are located at around $45^{\circ}$ with the mean flow. 


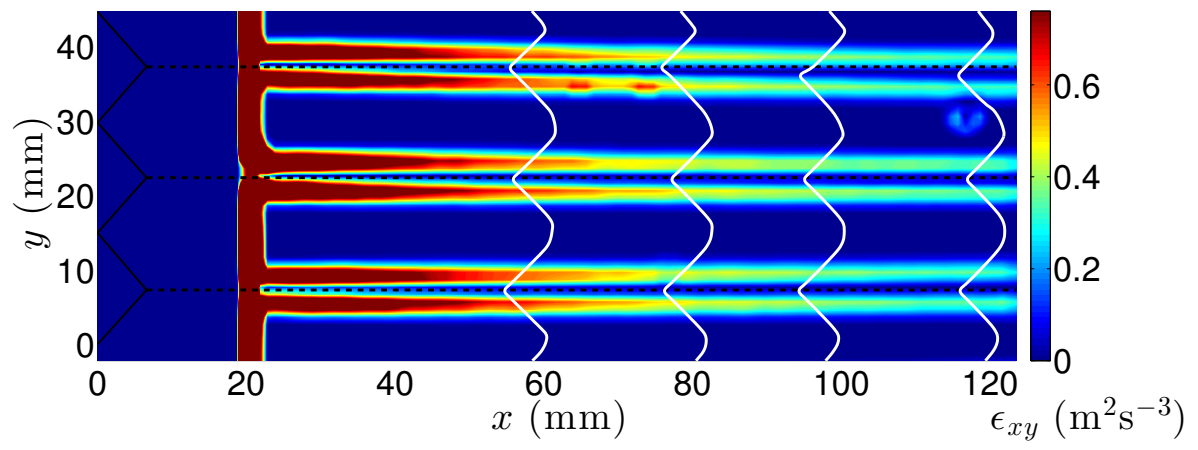

Figure 5: Contours of the viscous dissipation rate of kinetic energy $\left(\epsilon_{x y}\right)$ with the directions of stretching in white at $z=4 \mathrm{~mm}$ for $R e=2500$.

In Fig. 6, the angle between the stretching vectors and the streamwise direction $\vec{x}$ along $y$ are shown at different locations $x=43.4,118.7$ and $264.2 \mathrm{~mm}$ with the following respective symbols $*$, + and $\diamond$. The sketch of the saw-tooth cut at the flat leading edge are plotted in order to help understand the figure better. It can be shown that around the dotted line corresponding to the locations of the symmetry line of the pairs of counter rotating vortices as shown elsewhere, the angles of the stretching directions are located respectively at approximately $45^{\circ}$ and $-45^{\circ}$. For large $x$, such as $x=264.2 \mathrm{~mm}$, the angle slightly deviates further from $45^{\circ}$ indicating the break down of the counter-rotating vortices. It is interesting to note that the main direction of stretching located at around $45^{\circ}$ to the main flow is a feature of the so-called hairpin vortices (Davidson, 2004). This specific result for stretching is therefore similar to the one from the counter-rotating vortices studied in this current work. 


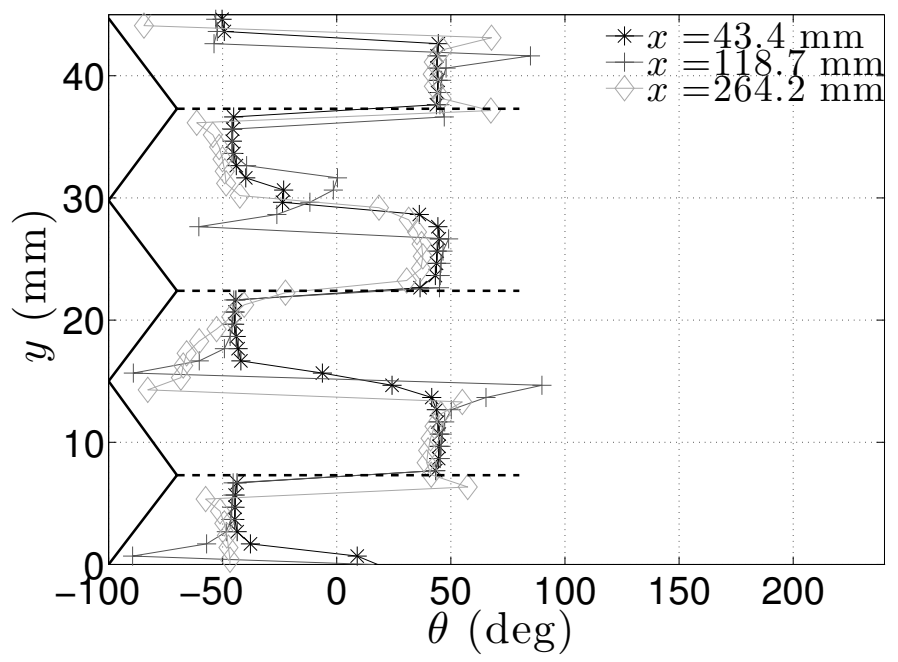

Figure 6: Angle between the stretching direction and the streamwise direction $\vec{x}(\theta)$ at $z=4 \mathrm{~mm}$ for $R e=2500$ at different streamwise locations: $x=43.4,118.7$ and $264.2 \mathrm{~mm}$.

\subsection{PIV results along the $(x, z)$ plane}

Particle Image Velocimetry (PIV) measurements have also been taken along the $(x, z)$ plane at $y=7.3 \mathrm{~mm}$ which corresponds to the upwash region where the boundary layer is less stable than in the downwash region and these measurements will supplement the $(x, y)$ plane

In order to check the validity of the PIV data between the $(x, z)$ and $(x$, $y$ ) planes, the evolutions of the streamwise velocity $u_{x}$ along the $x$-direction have been plotted in Fig. 7 along the lines where both planes match for $R e=2500$ for $z=7 \mathrm{~mm}$ and $y=7.3 \mathrm{~mm}$. In both plots, the ' + ' symbol represents the data along the $(x, y)$ plane while the star symbol represents the data for the $(x, z)$ plane. The data along both planes match with a 
maximum percentage error of $2 \%$.

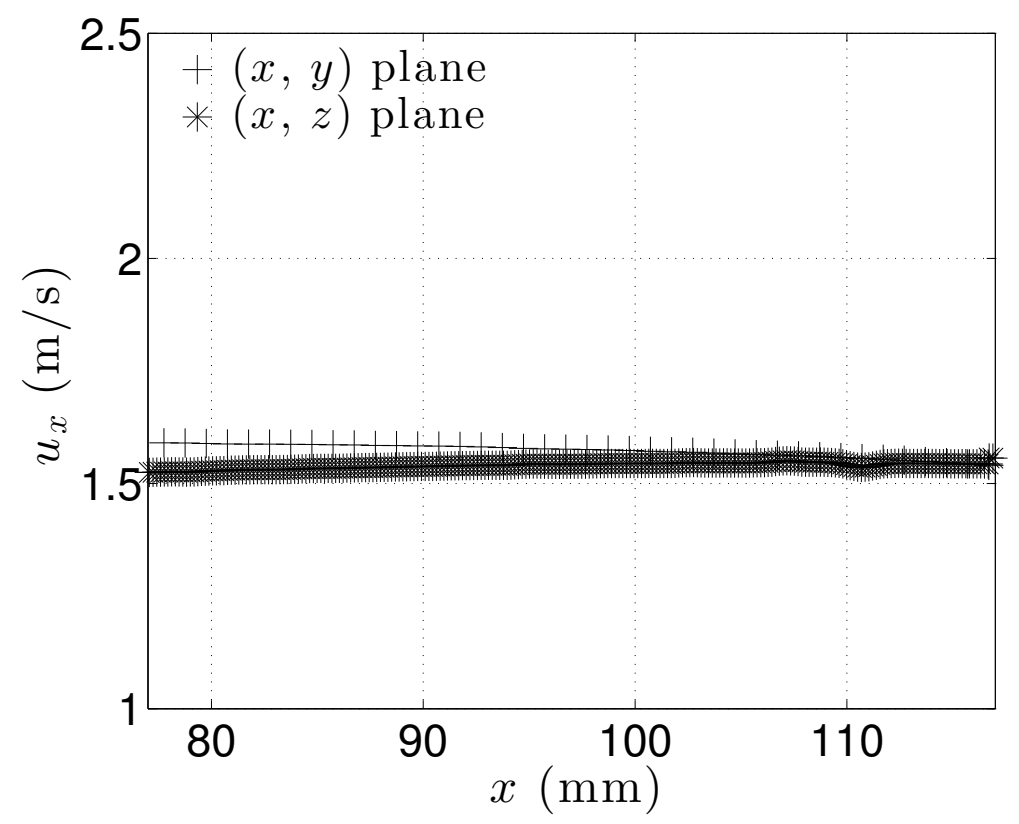

Figure 7: Comparison of the streamwise velocity $\left(u_{x}\right)$ at $z=7 \mathrm{~mm}$ between the $(x, y)$ plane and $(x, z)$ plane for $R e=2500$ and $y=7.3 \mathrm{~mm}$ (upwash region).

In Fig. 8, the vorticity contour $\omega_{y}$ in the upwash region $(y=7.3 \mathrm{~mm})$ is shown for $R e=2500$ up to the streamwise location of the second peak. The black line corresponds to the location where the streamwise velocity achieves $95 \%$ of the maximum velocity (for experimental reasons, 95\% was chosen instead of $99 \%$ to avoid any errors in determining the location), this location is related to the thickness of the boundary layer. On the plot, $\omega_{y}$ ranges from $-1500 \mathrm{~s}^{-1}$ to $200 \mathrm{~s}^{-1}$. Moreover, similarly to Fig. 2 (a) - (c), there were 2 parts within the field of view that were locally opaque where no velocity vectors could be obtained from PIV. These 2 areas are located between $x=0 \mathrm{~mm}$ and $6 \mathrm{~mm}$ and $x=116 \mathrm{~mm}$ and $135 \mathrm{~mm}$, they are blacked-out in Fig. 8 . 
It can be seen that over the flat plate part the boundary layer plotted with a black line has a positive slope suggesting the increase in size of the pair of counter-rotating vortices. Note that the vorticity of the vortices located above the valley between the two peaks at $x=188.2 \mathrm{~mm}$ and $x=264.2 \mathrm{~mm}$ is strictly negative. Thus, it shows that the vortices do not follow the borders of the wavy channels.

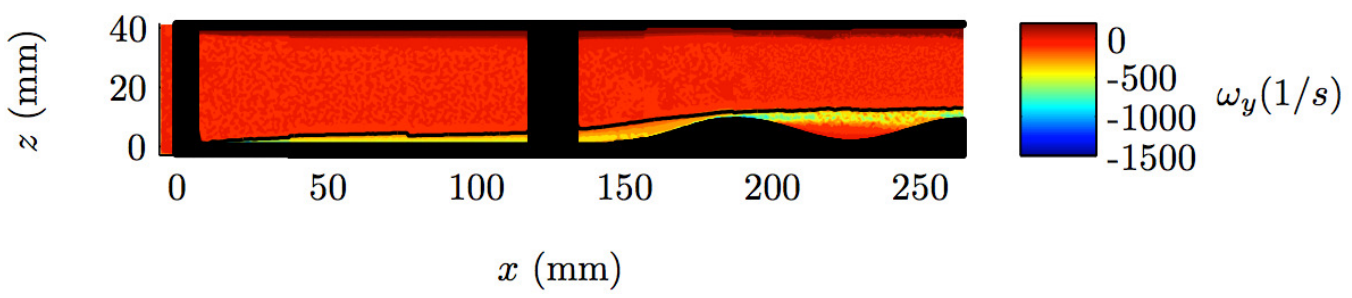

Figure 8: Spanwise vorticity $\left(\omega_{y}\right)$ contours along the $(x, z)$ plane for $R e=2500$ and $y=7.3 \mathrm{~mm}$ (upwash region).

The velocity vector plot for $R e=2500$ and at $y=7.3 \mathrm{~mm}$ (upwash region) is shown along the first valley in Fig. 9. Note that only one velocity vector for every five is shown along the $z$-direction in Fig. 9 in order to distinguish each velocity vector. Moreover, the modulus of the velocity field is squarerooted in order to magnify the size of the smaller vectors. The clockwise rotation of the velocity vectors indicates the appearance of a recirculation flow or reverse flow vortex in the valley which forms a separation bubble. This result is in a good agreement with the qualitative visualization results from Budiman et al. (2015).

Similarly to the discussion for the $(x, y)$ plane, mixing is also evaluated along the $(x, z)$ plane. The following two figures 10 and 11 (a) - (b) evaluate mixing from two different complementary approaches. 


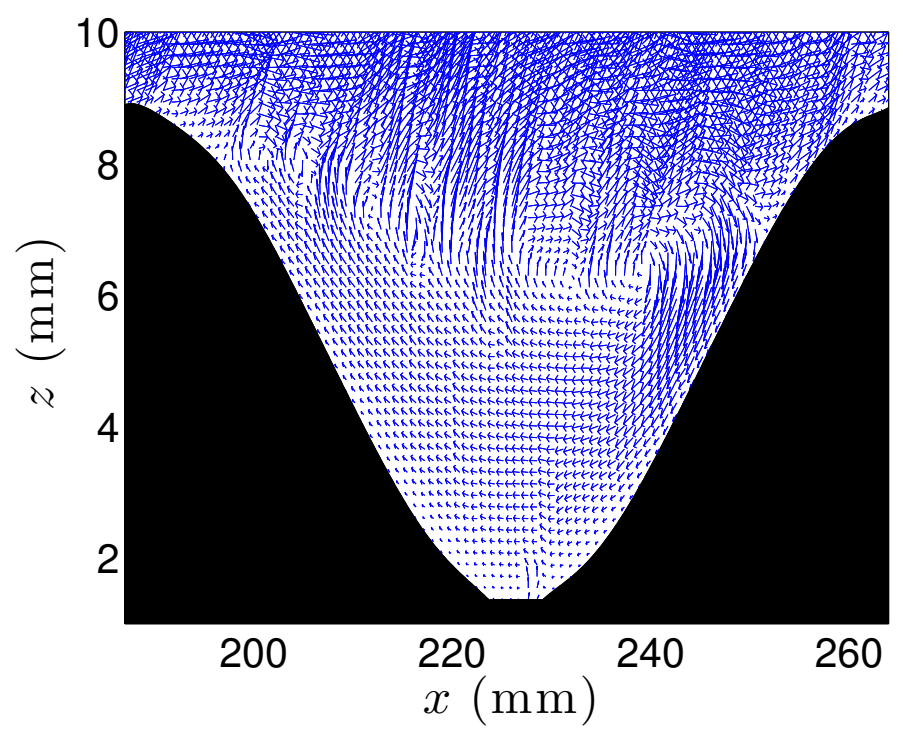

Figure 9: Velocity profile plot located in the first valley for $R e=2500$ and $y=7.3 \mathrm{~mm}$ (upwash region).

In Fig. 10, the evolution of $\overline{\epsilon_{x z}}$ averaged from the wall surface to the boundary layer location (shown with a black line in Fig. 8) is superimposed in grey along the streamwise direction for $R e=2500$ with $\epsilon_{x z}$ calculated in 2 dimensions along the $(x, z)$ plane. On the same figure, the wavy surface is plotted in black in order to compare the different values of $\overline{\epsilon_{x z}}$ with their streamwise locations over the wavy surface. The flat part lies up to $x=145$ $\mathrm{mm}$ with the part located between $x=116 \mathrm{~mm}$ and $135 \mathrm{~mm}$ being non-valid as explained in Fig. 8, therefore $\overline{\epsilon_{x z}}$ is null over this region. $\overline{\epsilon_{x z}}$ decreases from $x=12 \mathrm{~mm}$ with a value of $13.9 \mathrm{~m}^{2} \mathrm{~s}^{-3}$ to $0.85 \mathrm{~m}^{2} \mathrm{~s}^{-3}$ at $x=145 \mathrm{~mm}$. Therefore, the averaged viscous dissipation rate of kinetic energy is greatly reduced as the counter-rotating vortices move away from the entrance of the channel. 


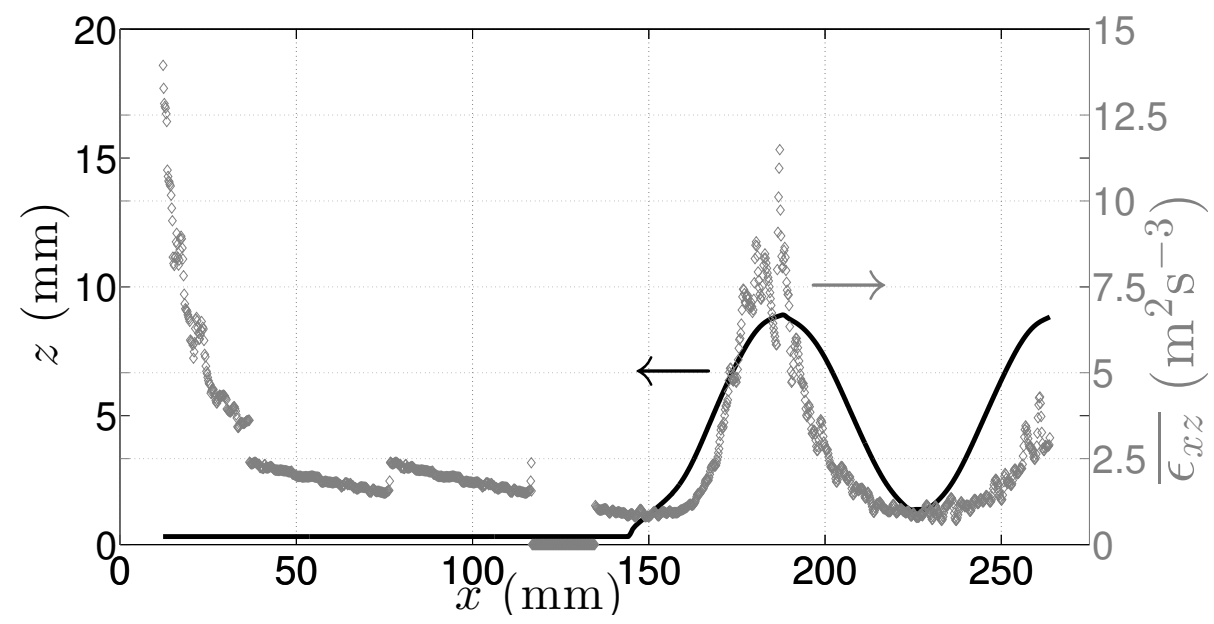

Figure 10: Evolution of the averaged viscous dissipation rate of kinetic energy $\left(\overline{\epsilon_{x z}}\right)$ in grey with the curvature of the wavy channel in black for $R e=2500$.

When the flow evolves from the flat part to the first peak at $x=188.2$ $\mathrm{mm}, \overline{\epsilon_{x z}}$ increases back to a local maximum of $11.6 \mathrm{~m}^{2} \mathrm{~s}^{-3}$, a value very close to its initial value in the entrance of the channel, before decreasing again from the first peak to the first valley at $x=226 \mathrm{~mm}$ to $0.82 \mathrm{~m}^{2} \mathrm{~s}^{-3}$. Finally, on the last part going up to the second peak $\overline{\epsilon_{x z}}$ increases to $4.3 \mathrm{~m}^{2} \mathrm{~s}^{-3}$. This value corresponds to an increase of more than 5 times the value of $\overline{\epsilon_{x z}}$ at the second valley streamwise location. Therefore, $\overline{\epsilon_{x z}}$ does increase while the flow goes from the valley to the peak locations and decrease when it travels from the peak to the valley locations and the surface parts. This approach shows that laminar mixing due to the counter-rotating streamwise vortices increases over favourable pressure gradients and decreases over flat and adverse pressure gradients.

The evolution of $\left(u_{x} / U_{\infty}\right)$ along $z / 2 a$ at different streamwise locations in the upwash region (at $y=7.3 \mathrm{~mm}$ ) for $R e=2500$ are shown in Fig. 11 (a) 
- (b) in which the origin of the vertical axis refers to the wall surface. The streamwise locations from $x=10 \mathrm{~mm}$ to $x=145 \mathrm{~mm}$ correspond to the flat part while the wavy part starts from $x=145 \mathrm{~mm}$ to $x=265 \mathrm{~mm}$. From each profile, it is interesting to calculate the wall shear stress as defined by:

$$
\tau_{w}=\mu\left(\frac{\partial u_{x}}{\partial z}\right)_{z=0}
$$

with $\mu$, the fluid dynamic viscosity. It has been shown that the calculation of the wall shear stress in the linear region corresponds to the region where $\mathrm{z}^{+}$is below 8 in wall units (Tandiono et al., 2009). $\mathrm{z}^{+}$is defined as:

$$
z^{+}=\frac{\sqrt{\frac{\left(\tau_{w}\right)_{z=0}}{\rho} z}}{\nu}
$$

with $\rho$, the volumic mass and $\nu$, the kinematic viscosity.

In Fig. 11 (a) - (b), the linear regions of the velocity profiles to calculate the wall shear stress are fitted with dotted lines using the least-square method. It can be seen that over the flat plate part, the wall shear stress becomes less and less important going from $\tau_{w}=0.0160 \mathrm{~Pa}$ at $x=10 \mathrm{~mm}$ to $0.0075 \mathrm{~Pa}$ at $x=90 \mathrm{~mm}$ and $0.0038 \mathrm{~Pa}$ at $x=145 \mathrm{~mm}$ as shown in Fig. 11 (a). When the counter rotating vortices travel from the first valley to the first peak of the wavy surface from $x=145 \mathrm{~mm}$ to $x=188 \mathrm{~mm}$, the wall shear stress increases to 0.0179 Pa, cf. Fig. 11 (a) - (b).

In Fig. 11 (b), the boundary velocity profiles along $z / 2 a$ are plotted from $x=188 \mathrm{~mm}$ to $x=265 \mathrm{~mm}$. The wall shear stress decreases from 0.0179 $\mathrm{Pa}$ at $x=188 \mathrm{~mm}$ to $-0.0006 \mathrm{~Pa}$ at $x=226 \mathrm{~mm}$ which corresponds to the location of the second valley with the presence of the reverse flow as indicated in Fig. 9. Finally, as the counter rotating vortices travel to the second peak 
located at $x=265 \mathrm{~mm}, \tau_{w}$ increases back to $0.0089 \mathrm{~Pa}$.

It is observed from Fig. 11 (a) - (b), that the wall shear stress from the counter-rotating vortices decreases over the flat part and when the flow goes from peak to valley while increasing when it is going from valley to peak. The increase of the wall shear stress as the vortices evolve from valley to peak could be due to the constriction of the channel and the centrifugal force from the surface curvature resulting in a "fuller" velocity profile; whereas from peak to valley, the presence of a separation bubble affects the wall shear stress value. Therefore, the mixing due to the wall shear stress decreases over the flat and adverse pressure gradient parts while increases when travelling over the favourable pressure gradient part.

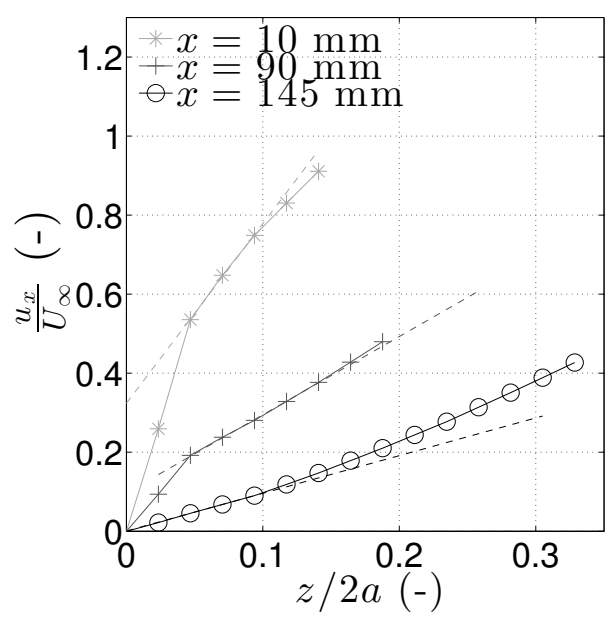

(a)

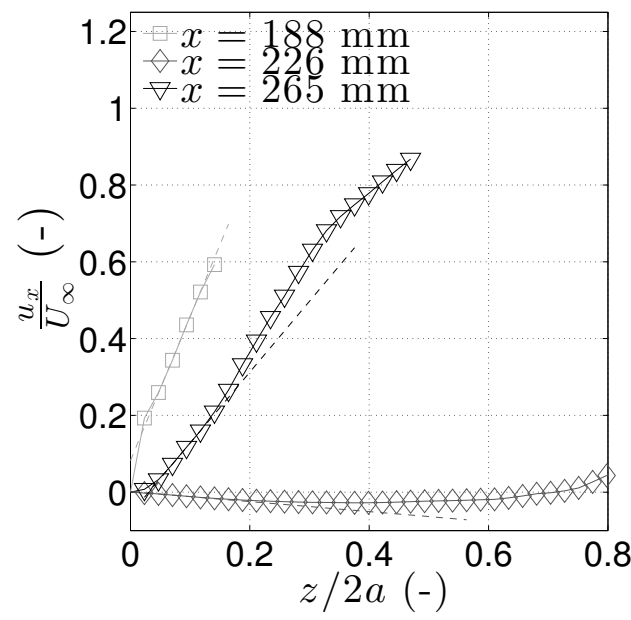

(b)

Figure 11: Evolution of normalised mean velocity profiles $\left(u_{x} / U_{\infty}\right)$ along $z / 2 a$ at different streamwise locations $(x)$ for $y=7.3 \mathrm{~mm}$ and $R e=2500$ : (a) from $x=10$ to $145 \mathrm{~mm}$; (b): from $x=188$ to $265 \mathrm{~mm}$. 


\section{Conclusions}

Counter-rotating streamwise vortices with uniform spanwise wavelengths inside a flat rectangular channel with one-sided wavy wall have been characterized using Particle Image Velocimetry (PIV) measurements for $R e=2500$. Two other Re values of 2885 and 3333 have also been considered for quantification purpose. This study was realised along streamwise and spanwise planes of measurement. Several conclusions can be made from this study. It has been shown experimentally that pairs of counter-rotating vortices are centered along the spanwise directions of the saw-tooth trough $\left(\omega_{z}=0\right)$. For a constant Reynolds number at a fixed normal distance from the surface, the vorticity of the counter-rotating streamwise vortices $\omega_{z}$ decreases as they evolve downstream, while it increases as the Reynolds number increases. The PIV data also reveal that the counter-rotating streamwise vortices are getting closer to each other when evolving downstream as they grow in size while their vorticity levels, $\omega_{z}$, decrease. While for the mixing, it is evident that at a normal distance of $z=4 \mathrm{~mm}$ from the surface, the viscous dissipation rate of kinetic energy decreases along the streamwise direction $\vec{x}$ over the flat part with the main direction of stretching oriented at about 45 degrees to the streamwise direction alike hairpin vortices. It has been equally

shown from the $(x, z)$ plane that the averaged viscous dissipation rate of kinetic energy due to the counter-rotating streamwise vortices increases over favourable pressure gradients and decreases over flat and adverse pressure gradients. The mixing was also quantified from the wall shear stress of these vortices. The decrease of the mixing due to the downstream evolution of the wall shear stress of the counter-rotating streamwise vortices is evident as 
the vortices evolve along the flat plate part of the channel. On the contrary, the occurrence of the favorable pressure gradient when the flow travels from the flat part to the first peak and from the first valley to the second peak of the corrugation increases the mixing due to the wall shear stress of the counter-rotating streamwise vortices. 


$\begin{array}{ll}\text { Nomenclature } & \\ \epsilon & \text { Viscous dissipation rate of kinetic energy } \\ \theta & \text { Angle between the stretching vector and the streamwise } \\ \lambda & \text { direction } \\ \mu & \text { Wavelength of the wavy surface } \\ \nu & \text { Fluid dynamic viscosity } \\ \rho & \text { Fluid kinematic viscosity } \\ \tau_{w} & \text { Volumic mass } \\ \omega_{i} & \text { Wall shear stress } \\ \Omega_{i j} & \text { Vorticity along the } i_{t h} \text { direction } \\ a & \text { Rate-of-rotation tensor } \\ H & \text { Amplitude of wavy surface } \\ R e & \text { Channel gap, measured at the leading edge } \\ S & \text { Reynolds number }\left(\frac{1}{2} \frac{U_{\infty} H}{\nu}\right) \\ S_{i j} & \text { Ratio of amplitude of wavy surface to channel gap } \\ S_{11}^{*}, S_{22}^{*}, S_{33}^{*} & \text { Rate-of-strain tensor } \\ u_{i} & \text { Eigenvalues of rate-of-strain tensor } \\ U_{\infty} & \text { Velocity along the } i_{t h} \text { direction } \\ x, y, z & \text { Free-stream streamwise velocity } \\ z^{+} & \text {Cartesian coordinate system } \\ & \text { Dimensionless wall distance }\left(\frac{\sqrt{\frac{(\tau w)_{z=0}}{\rho} z}}{\nu}\right) \\ & \end{array}$

Acknowledgements The authors express their appreciation to Mr. Lucius Sim and Mr. M. N. Adha b Jamaludin for their help in setting up the 
experimental rig and in collecting the experimental data.

\section{References}

Aider, J. L., Duriez, T., Wesfreid, J. E., 2009. From natural to forced counterrotating steamwise vortices in boundary layers. Journal of Physics: Conference Series (137).

Asai, M., Floryan, J. M., 2006. Experiments on the linear instability of flow in a wavy channel. European Journal of Mechanics B/Fluids 25, 971-986.

Bahaidarah, H. M. S., Anand, N. K., Chen, H. C., 2005. Numerical study of heat and momentum transfer in channels with wavy walls. Numerical Heat Transfer, Part A 47, 417-439.

Bakchinov, A. A., Grek, G. R., Klingmann, B. G. B., Kozlov, V. V., 1995. Transition experiments in a boundary layer with embedded streamwise vortices. Physics of Fluids 7, 820-832.

Bouremel, Y., Yianneskis, M., Ducci, A., 2009. On the utilisation of vorticity and strain dynamics for improved analysis of stirred processes. Chemical Engineering Research and Design 87, 377-385.

Budiman, A. C., Mitsudharmadi, H., Bouremel, Y., Winoto, S. H., Low, H. T., 2015. Visualization of pre-set vortices in boundary layer flow over wavy surface in rectangular channel. Journal of Visualization 18, 669-677.

Budiman, A. C., Mitsudharmadi, H., Bouremel, Y., Winoto, S. H., Low, H. T., 2016. Development of pre-set counter-rotating streamwise vortices in wavy channel. Experimental Thermal and Fluid Science 71, 77-85. 
Clauser, M., Clauser, F., 1937. The effect of curvature on transition from laminar to turbulent boundary layer. NACA TN 613.

Davidson, P. A., 2004. Turbulence, An Introduction for Scientists and Engineers. Oxford University Press.

Floryan, J. M., 2002. Centrifugal instability of couette flow over a wavy wall. Physics of Fluids 14 (1), 312-322.

Floryan, J. M., 2007. Three-dimensional instabilities of laminar flow in a rough channel and the concept of hydraulically smooth wall. European Journal of Mechanics B/Fluids 26 (3), 305-329.

Gschwind, P., Regele, A., Kottke, V., 1995. Sinusoidal wavy channels with Taylor-Goertler vortices. Experimental Thermal and Fluid Science 11, 270-275.

H.Görtler, 1954. On the three-dimensional instability of laminar boundary layers on concave walls. NACA Technical Memorandum 13751375.

Mitsudharmadi, H., Winoto, S. H., Shah, D. A., 2004. Development of boundary-layer flow in the presence of forced wavelength Görtler vortices. Physics of Fluids 16 (11), 3983-3996.

Mitsudharmadi, H., Winoto, S. H., Shah, D. A., 2006. Development of most amplified wavelength Görtler vortices. Physics of Fluids 18, 014101-1.

Nishimura, T., Kajimoto, Y., Kawamura, Y., 1986. Mass transfer enhancement in channels with a wavy wall. Journal of Chemical Engineering of Japan 19, 142-144. 
Nishimura, T., Ohori, Y., Kajimoto, Y., Kawamura, Y., 1985. Mass transfer characteristics in a channel with symmetric wavy wall for steady flow. Journal of Chemical Engineering of Japan 18, 550-555.

Nishimura, T., Yano, K., Yoshino, T., Kawamura, Y., 1990. Occurrence and structure of Taylor-Goertler vortices induced in two-dimensional wavy channels for steady flow. Journal of Chemical Engineering of Japan 23 (6), 697-703.

Smith, A. M. O., 1955. On the growth of Taylor-Görtler vortices along highly concave walls. Q. Appl. Math. 13, 233.

Tandiono, T., Winoto, S. H., Shah, D. A., 2009. Wall shear stress in Görtler vortex boundary layer flow. Physics of Fluids 21, 084106-1.

Tandiono, T., Winoto, S. H., Shah, D. A., 2013. Spanwise velocity component in nonlinear region of Görtler vortices. Physics of Fluids 25, 104104.

Winoto, S. H., Crane, R. I., 1980. Vortex structure in laminar boundarylayers on a concave wall. Int. J. of Heat and Fluid Flow 2 (4), 221-231.

Winoto, S. H., Tandiono, T., Shah, D. A., Mitsudharmadi, H., 2011. Concave surface boundary layer flows in the presence of streamwise vortices. International Journal of Fluid Machinery and Systems 4 (1), 33-46. 